\title{
Biological interactions of CYP2C19 genotypes with CYP3A4*18, CYP3A5*3, and MDR1-3435 in living donor liver transplantation recipients
}

King-Wah Chiu ${ }^{1,2^{*}}$, Tsung-Hui Hu ${ }^{2}$, Toshiaki Nakano ${ }^{2}$, Kuang-Den Chen ${ }^{2}$, Chia-Yun Lai ${ }^{2}$, Li-Wen Hsu ${ }^{2}$, Hui-Peng Tseng ${ }^{2}$, Ho-Ching Chiu ${ }^{2}$, Yu-Fan Cheng ${ }^{2}$, Shigeru Goto ${ }^{2}$ and Chao-Long Chen ${ }^{1,2^{*}}$

\begin{abstract}
Background: Polymorphisms in CYP2C19 are related to the metabolic oxidation of drugs to varying degrees. The CYP3A4*18, CYP3A5*3, and MDR1-3435 variant alleles are very important, particularly in tacrolimus metabolism in organ transplant rejection.

Aim: The aim of this study is o explore possible interactions among different CYP2C19 genotypes, namely, between homozygous extensive metabolizers (HomEM), heterozygous extensive metabolizers (HetEM), and poor metabolizers (PM), and the CYP3A4*18, CYP3A5*3, and MDR1-3435 variants in living donors and patients who received a living donor liver transplant (LDLT).

Methods: This prospective study enrolled 133 living donors and 133 corresponding recipients. On the basis of the HomEM, HetEM, and PM CYP2C19 genotypes, the distributions of CYP3A4*18 (exon 10; T878C), CYP3A5*3 (intron 3; A6986G), and MDR1-3435 (exon 26; C3435T) genotypes were analyzed for single nucleotide polymorphisms among donors and recipients.

Results: Among 102 HomEM genotypes, including 56 donors and 46 recipients, 91.2\% of individuals harbored the T/T genotype of CYP3A4*18; 53.9\% possessed G/G, and 34.3\% had A/G genotypes of CYP3A5*3; and 38.2\% had C/C and $50.0 \%$ had $C / T$ genotypes at MDR1-3435. Among 130 HetEM genotypes, including 58 donors and 72 recipients, $97.7 \%$ of individuals possessed T/T genotype at CYP3A4* $18 ; 50.0 \%$ harbored $\mathrm{G} / \mathrm{G}$ and $41.5 \%$ had $\mathrm{A} / \mathrm{G}$ genotypes at CYP3A5*3; and 40.0\% had C/C and 49.2\% had C/T genotypes at MDR1-3435. In 34 PMs, including 19 donors and 15 recipients, 88.2\% had T/T genotypes at CYP3A4*18; $41.2 \%$ had G/G and 58.8\% had A/G genotypes at CYP3A5*3; and 47.1\% possessed C/C and $47.1 \%$ had C/T genotypes at MDR1-3435. On the basis of the CYP2C19 genotypes, no statistically significant distribution of genotypes were observed between donors and recipients for all genotypes of CYP3A4*18, CYP3A5*3, and MDR1-3435 ( $P>0.05)$.
\end{abstract}

Conclusions: In conclusion, the CYP2C19 genotypes do not affect the expression of CYP3A4*18, CYP3A5*3, or MDR13435 variants, which are independently distributed among donors and recipients during LDLT.

Keywords: Living donor liver transplantation, Cytochrome P450, CYP3A4*18, CYP3A5*3, MDR1-3435, CYP2C19 genotypes

\footnotetext{
* Correspondence: c471026@ms6.hinet.net; clchen@cgmh.org.tw

'Liver Transplant Program, Kaohsiung Chang Gung Memorial Hospital, Chang Gung University, College of Medicine, Kaohsiung, Taiwan

${ }^{2}$ Kaohsiung Chang Gung Memorial Hospital, Chang Gung University College of Medicine, 123 Ta-Pei Road, Niao-Sung, Kaohsiung 833, Taiwan
} 


\section{Introduction}

Cytochrome P450 in the liver is one of the key enzyme complexes in the primary drug-metabolizing system in humans. Recipients of living-donor liver transplantation (LDLT) exhibit interesting biological distributions of CYP2C19 genotypes that differ between the recipient's tissue and the newly grafted tissue [1]. Acute rejection or abnormal postoperative liver function after LDLT could result from complications in cytochrome P450 function $[2,3]$. We have recently reported a homogenous phenomenon observed in CYP2C19 genotypes [4]. Antirejection agents such as tacrolimus usually target CYP3A4, CYP3A5, and MDR-1, which are the major metabolic isoenzymes of cytochrome P450. In the present study, we aimed to investigate the CYP2C19 genotypes, which have been classified as homozygous extensive metabolizers (HomEM), heterozygous extensive metabolizers (HetEM), and poor metabolizers (PM), and to identify any interactions between the CYP3A4*18, CYP3A5*3, and MDR13435 variants by characterizing differences between the genotype distribution of healthy living donors and patients with liver disease who received LDLT.

\section{Methods}

This present study included 133 living donors and 133 consecutive LDLT recipients who were enrolled in our transplantation program. The mean age of the recipients was 42.62 years (range, 0.6 to 68 years), including 98 male and 35 female subjects. The etiologies for LDLT are shown in Table 1. There were 60 (45.1\%) recipients with hepatitis B-related end-stage liver disease (ESLD), of which 35 (58.3\%) were associated with hepatocellular carcinoma (HCC); 22 (16.5\%) recipients with hepatitis C-related ESLD, including 11 (50.0\%) with HCC; 9 (6.8\%) recipients with both hepatitis B- and C-related ESLD, including 5 (55.6\%) with HCC; 8 (6.0\%) recipients with alcoholic-related ESLD, including 2 (25\%) with HCC; and 23 pediatric recipients with biliary atresia. The remaining

Table 1 Etiology of the 133 study subjects who required living donor liver transplantation

\begin{tabular}{lcc}
\hline Category & $\begin{array}{c}\text { Recipient } \\
\text { N (\%) }\end{array}$ & $\begin{array}{c}\text { Associated with HCC } \\
\text { N (\%) }\end{array}$ \\
\hline HBV & $60(45.1)$ & $35(58.3)$ \\
HCV & $22(16.5)$ & $11(50.0)$ \\
HBV + HCV & $9(6.8)$ & $5(55.6)$ \\
Alcoholic & $8(6.0)$ & $2(25.0)$ \\
Biliary atresia & $23(17.3)$ & \\
Other $^{\text {a }}$ & $11(8.3)$ & \\
Total $^{-133(100)}$ & $53(39.9)$ \\
\hline
\end{tabular}

autoimmune (1), Alagille syndrome (3), portal vein occlusion (1), primary biliary cirrhosis (2), polycystic liver (1), and cryptogenic liver cirrhosis (3). $\mathrm{HBV}$, hepatitis B virus; HCC, hepatocellular carcinoma; HCV, hepatitis $C$ virus.
11 recipients had autoimmune liver disease (1 patient), Alagille syndrome (3 patients), portal vein occlusion (1 patient), primary biliary cirrhosis (2 patients), polycystic liver (1 patient), or cryptogenic liver cirrhosis (3 patient). Complete liver function was assessed on day 1 after liver transplantation (D1) and on day 30 after liver transplantation (D30), including measurements of alanine transferase (ALT), aspartate transferase (AST), total bilirubin (T-Bil), prothrombin time (PT) with international normal range (INR), and albumin (Alb). Donors and recipients were classified on the basis of the CYP2C19 genotype, as detailed in our previous report [1]. HomEMs were individuals with wild-type alleles (genotype: "1/"1), whereas HetEMs were those who possessed a single mutated allele (genotype: *1/"2 or "1/*3). Patients with homozygous mutations ( $\mathrm{m} 1$ in exon 5 or $\mathrm{m} 2$ in exon 4) of CYP2C19 (genotype: " $2 /{ }^{*} 2$, " $3 / * 3$, or *2/*3) were defined as PMs. This study comprised a total of 102 HomEMs, including 56 donors and 46 recipients; 130 HetEMs, including 58 donors and 72 recipients; and $34 \mathrm{PMs}$, including 19 donors and 15 recipients. On the basis of the CYP2C19 genotype classification, HomEM, HetEM, or PM individuals were analyzed to test for carrier status of other known single nucleotide polymorphism (SNP) variant alleles to determine biological distributions of CYP3A4*18 (exon 10; T878C), CYP3A5*3 (intron 3; A6986G), and MDR1-3435 (exon 26; C3435T). Genomic DNA was extracted from peripheral leukocytes and genotyped for evaluating the CYP2C19, CYP3A4*18, CYP3A5*3, and MDR1-3435 status by polymerase chain reaction-restriction fragment length polymorphism (PCR-RFLP) and direct sequencing analysis.

\section{Genotyping of CYP3A4, CYP3A5, and MDR1-3435}

Polymerase chain reaction/ligase detection reaction assay (PCR/LDR) was employed for genotyping the CYP3A4*18B and CYP3A5*3 SNPs. The PCR conditions consisted of a denaturation step at $95^{\circ} \mathrm{C}$ for $15 \mathrm{~min}$, followed by 35 cycles of $94^{\circ} \mathrm{C}$ for $30 \mathrm{~s}, 65^{\circ} \mathrm{C}$ for $1 \mathrm{~min}$, and $72^{\circ} \mathrm{C}$ for $1 \mathrm{~min}$, followed by a final extension step at $72^{\circ} \mathrm{C}$ for $7 \mathrm{~min}$. The specific amplified fragments were used in an LDR assay to identify the mutations associated with $C Y P 3 A 4 * 18 B$ and CYP $3 A 5 * 3$. The LDR assay was performed as follows: 10 $\mu \mathrm{L}$ of the reaction mix contained $1 \mu \mathrm{L}$ of $1 \times$ ligase reaction buffer (New England Biolabs, USA), $1 \mu \mathrm{L}$ of probes (12.5 $\mathrm{pmol} / \mu \mathrm{L}$ each), $0.05 \mu \mathrm{L}(2 \mathrm{U})$ of thermostable Taq DNA ligase (New England Biolabs), and $1 \mu \mathrm{L}$ of PCR product. The ligation reaction was performed with a GeneAmp PCR System 9600 (Perkin Elmer, USA) as follows: $15 \mathrm{~min}$ at $95^{\circ} \mathrm{C}$, followed by 35 cycles of $30 \mathrm{~s}$ at $94^{\circ} \mathrm{C}$ and $2 \mathrm{~min}$ at $60^{\circ} \mathrm{C}$. The products were separated by agarose gel electrophoresis and analyzed with an ABI PRISM 377 DNA sequencer [5]. Genotyping was performed using an independent external contractor (Biowing Applied 
Biotechnology Co. Ltd., China). Genomic DNA was isolated from whole blood using the UltraPure ${ }^{\mathrm{rM}}$ Genomic DNA Isolation Kit (Shanghai SBS Genetech Technology Co., China). PCR-RFLP was performed to genotype exon 26 (C3435T) variant alleles in the MDR1 gene, with slight modifications. The PCR conditions consisted of a denaturation step at $95^{\circ} \mathrm{C}$ for $5 \mathrm{~min}$, followed by 30 cycles of denaturation at $94^{\circ} \mathrm{C}$ for $30 \mathrm{~s}$, annealing at $54^{\circ} \mathrm{C}$ to $59^{\circ} \mathrm{C}$ for $50 \mathrm{~s}$, and elongation at $72^{\circ} \mathrm{C}$ for $1 \mathrm{~min}$, followed by a final extension at $72^{\circ} \mathrm{C}$ for $10 \mathrm{~min}$. PCR products were digested with DpnII (C3435T) and analyzed by electrophoretic separation on agarose gels, followed by direct visualization over an ultraviolet transilluminator after ethidium bromide staining [6].

This work was supported by a grant from Chang Gung Memorial Hospital (CMRPG8A0631 to K-WC) of Taiwan, which also granted ethical approval to our study. This study was approved by the Institutional Review Board (IRB), and informed consent was obtained from participants or from a parent or guardian in case of minor participants.

\section{Statistical analyses}

Statistical analyses were performed using SPSS software (version 12.0; SPSS, Chicago, IL, USA). Parameters between HomEM or HetEM and PMs in donors and recipients were compared using the $X^{2}$ test, Fisher's exact test, and Student's $t$-test. $P$ values less than 0.05 were considered statistically significant.

\section{Results}

In this study, 68.4\% (91/133) of ESLD cases were virusrelated, including $45.1 \%(60 / 133)$ of hepatitis B cases, $16.5 \%(22 / 133)$ of hepatitis C cases, and 6.8\% (9/133) of cases with both hepatitis B and C. Furthermore, 39.9\% (53/133) recipients had HCC. Excluding the 23 biliary atresia and 3 Alagille syndrome pediatric cases, $49.5 \%$
(53/107) of adult recipients had HCC (Table 1). Clinical profiles of the 133 donors and 133 recipients on the day of the LDLT operation are shown in Table 2. The mean age of the donor population was significantly younger than the recipient population (30.27 years (range 18 to 53 years) versus 42.62 years (range 0.6 to 68 years); $P<0.001$ ). On D1 of liver transplantation, all donors showed significantly better results of all liver function tests than did the recipients, including ALT (14.43 \pm 14.75 versus $252.35 \pm$ 446.40; $P<0.001)$, AST $(18.3 \pm 7.19$ versus $292.42 \pm 660.86$; $P<0.001)$, T-Bil $(0.64 \pm 0.57$ versus $7.19 \pm 11.71 ; P<0.001)$, PT (INR) (0.97 \pm 0.09 versus $1.91 \pm 3.34 ; P=0.003)$, and Alb $(4.24 \pm 0.79$ versus $3.06 \pm 0.88 ; P<0.001)$. On D30, after LDLT, all liver function tests of the recipients were significantly better than those on D1, including ALT (252.43 \pm 446.40 versus $82.40 \pm 244.43, P<0.001)$, AST $(292.42 \pm$ 660.86 versus $65.54 \pm 300.20, P<0.001)$, T-Bil $(7.19 \pm 11.71$ versus $1.24 \pm 3.60, P<0.001)$, PT (INR) $(1.91 \pm 3.34$ versus $1.05 \pm 0.40, P<0.001)$, and Alb $(3.06 \pm 0.88$ versus $3.74 \pm$ $0.95, P<0.001)$.

On the day of LDLT, 107 adult recipients received tacrolimus and 26 pediatric recipients received cyclosporine A (CsA). The serum concentrations of tacrolimus and CsA were $2.51 \pm 2.73 \mathrm{ng} / \mathrm{mL}$ and $283.89 \pm 308.93$ $\mathrm{ng} / \mathrm{mL}$, respectively, on $\mathrm{D} 1$ and $6.17 \pm 9.58 \mathrm{ng} / \mathrm{mL}$ and $1058.30 \pm 582.37 \mathrm{ng} / \mathrm{mL}$, respectively, on D30; these results show that the serum concentrations of both tacrolimus and cyA were significantly higher on D30 than on D1 after LDLT $(P<0.001$; Table 2$)$.

Among the 266 subjects with CYP2C19 genotypes (comprising 133 healthy donors and 133 diseased recipients), 102 had HomEM genotypes, including 56 donors and 46 recipients. The 2 wild-type CYP3A4*18 genotypes, $\mathrm{T} / \mathrm{T}$ and $\mathrm{T} / \mathrm{C}$ genotypes, were distributed as follows: $53.8 \%$ (50/93) of individuals with the $\mathrm{T} / \mathrm{T}$ genotype were donors and $46.2 \%(43 / 93)$ were recipients, and $66.7 \%(6 / 9)$ of cases with the T/C genotype were donors

Table 2 Clinical profiles of 133 donors and 133 recipients for living donor liver transplantation and comparison of their clinical profiles on day 1 (D1) and day 30 (D30) after transplantation

\begin{tabular}{|c|c|c|c|c|c|c|}
\hline \multirow{2}{*}{$\begin{array}{l}\text { Category } \\
\text { Age (mean) (range) }\end{array}$} & \multirow{2}{*}{$\begin{array}{l}\text { Donor } \mathbf{N}=\mathbf{1 3 3} \\
30.27(18 \text { to } 53)\end{array}$} & \multicolumn{2}{|c|}{ Recipient $N=133$} & \multicolumn{3}{|c|}{$P$ value } \\
\hline & & & -68) & & $<0.001$ & \\
\hline \multirow[t]{2}{*}{ Sex M:F } & \multirow[t]{2}{*}{$83: 50$} & \multicolumn{2}{|c|}{$98: 35$} & \multicolumn{3}{|c|}{0.292} \\
\hline & & D1 & D30 & $\mathrm{D}: \mathrm{RD} 1$ & D:RD30 & $\mathrm{RD1}: \mathrm{RD} 30$ \\
\hline ALT & $14.43 \pm 14.75$ & $252.35 \pm 446.40$ & $82.40 \pm 244.43$ & $<0.001$ & 0.003 & $<0.001$ \\
\hline AST & $18.3 \pm 7.19$ & $292.42 \pm 660.86$ & $65.54 \pm 300.20$ & $<0.001$ & 0.087 & $<0.001$ \\
\hline T-Bil & $0.64 \pm 0.57$ & $7.19 \pm 11.71$ & $1.24 \pm 3.60$ & $<0.001$ & 0.071 & $<0.001$ \\
\hline PT (INR) & $0.97 \pm 0.09$ & $1.91 \pm 3.34$ & $1.05 \pm 0.40$ & 0.003 & 0.025 & $<0.001$ \\
\hline Alb & $4.24 \pm 0.79$ & $3.06 \pm 0.88$ & $3.74 \pm 0.95$ & $<0.001$ & 0.148 & $<0.001$ \\
\hline \multicolumn{2}{|c|}{ Tacrolimus (ng/mL) (n = 107) } & $2.51 \pm 2.73$ & $6.17 \pm 9.58$ & & & $<0.001$ \\
\hline \multicolumn{2}{|l|}{ cyA (ng/mL) $(n=26)$} & $283.89 \pm 308.93$ & $1058.30 \pm 582.37$ & & & $<0.001$ \\
\hline
\end{tabular}

$\mathrm{D}$, donor; D1, post liver transplantation day 1; D30, post liver transplantation day 30; R, recipient. 
and $33.3 \%(3 / 9)$ were recipients. The $3 C Y P 3 A 5 * 3$ genotypes namely, G/G, A/G, and A/A were distributed as follows: $58 \%(32 / 55)$ of individuals with the G/G genotype were donors and $42.0 \%(23 / 55)$ were recipients, $48.6 \%(17 / 35)$ of individuals with the A/G genotype were donors and 51.4\% (18/35) were recipients, and 58.3\% (7/ 12) of individuals with the A/A genotype were donors and $41.7 \%(5 / 12)$ were recipients. The 3 MDR1-3435 genotypes, $\mathrm{C} / \mathrm{C}, \mathrm{C} / \mathrm{T}$, and $\mathrm{T} / \mathrm{T}$, were distributed as follows: $59.0 \%(23 / 39)$ of individuals with the $\mathrm{C} / \mathrm{C}$ genotype were donors and $41.0 \%(16 / 39)$ were recipients, $56.9 \%(29 / 51)$ of individuals with the $\mathrm{C} / \mathrm{T}$ genotype were donors and $43.1 \%(22 / 51)$ were recipients, and $33.3 \%(4 / 12)$ of individuals with the $\mathrm{T} / \mathrm{T}$ genotype were donors and $66.7 \%$ $(8 / 12)$ were recipients. There was no statistically significant correlation between healthy donors and diseased recipients bearing the $C Y P 2 C 19$ HomEM genotype with $C Y P 3 A 4 * 18, C Y P 3 A 5 * 3$, or $M D R 1-3435$ polymorphisms $(P>0.05)$.

In this study, 130 individuals had the HetEM genotype, including 58 donors and 72 recipients. The 2 wild-type and $C Y P 3 A 4 * 18$ genotypes were distributed as follows: $45.7 \%(58 / 127)$ of individuals with the T/T genotype were donors and $54.3 \%(69 / 93)$ were recipients, and $0 \%$ $(0 / 3)$ of those with the $\mathrm{T} / \mathrm{C}$ genotype were donors and $100.0 \%$ (3/3) were recipients. The 3 CYP $3 A 5 * 3$ genotypes were distributed as follows: $46.2 \%(30 / 65)$ of individuals with the $\mathrm{G} / \mathrm{G}$ genotype were donors and $53.8 \%$ (35/65) were recipients, $48.0 \%(26 / 54)$ of individuals with the A/G genotype were donors and $52.0 \%$ (28/54) were recipients, and $18.2 \%(2 / 11)$ of individuals with the A/A genotype were donors and $81.8 \%$ (9/11) were recipients. The 3 MDR1-3435 genotypes were distributed as follows: $42.3 \%(22 / 52)$ of individuals with the $\mathrm{C} /$ $\mathrm{C}$ genotype were donors and $57.7 \%(30 / 52)$ were recipients, $50.0 \%(32 / 64)$ of individuals with the $\mathrm{C} / \mathrm{T}$ genotype were donors and $50.0 \%(32 / 64)$ were recipients, and $28.6 \%(4 / 14)$ of individuals with the $\mathrm{T} / \mathrm{T}$ genotype were donors and $71.4 \%(10 / 14)$ were recipients. No statistically significant association was observed between healthy donors and diseased recipients with the CYP2C19 HetEM genotype and the CYP3A4*18,CYP3A5*3, or MDR1-3435 genotypes $(P>0.05)$. The data presented in Table 3 summarizes the negative correlations between the HomEM, HetEM, and PM CYP2C19 genotypes and the CYP3A4*18, $C Y P 3 A 5 * 3$, or $M D R 1-3435$ genotypes. Our data indicate that these genotypes had similar distributions in both donors and recipients, suggesting that there were no statistically significant differences between CYP3A4*18 (T/T and T/C), CYP3A5*3 (G/G, A/G and A/A), and MDR1-3435 $(\mathrm{C} / \mathrm{C}, \mathrm{C} / \mathrm{T}$ and $\mathrm{T} / \mathrm{T})$ haplotypes, as well as the different CYP2C19 HomEM, HetEM, and PM genotypes between healthy donors and recipients with ESLD. Hence, there were independent isoenzymes and no correlation of genetic interaction between CYP2C19 and CYP3A4*18, $C Y P 3 A 5 * 3$, or MDR1-3435 not only, but also the variant haplotypes or genotypes.

There were 34 PMs in this cohort, including 19 donors and 15 recipients. The 2 wild-type CYP3A4*18 genotypes were distributed as follows: $3.3 \%(16 / 30)$ of individuals with the $\mathrm{T} / \mathrm{T}$ genotype were donors and $46.7 \%$ $(14 / 30)$ were recipients, $75.0 \%(3 / 4)$ of individuals with the $\mathrm{T} / \mathrm{C}$ genotype were donors and $25.0 \%(1 / 4)$ were recipients. Only 2 of the possible $C Y P 3 A 5 * 3$ genotypes were

Table 3 Association between the CYP2C19 genotypes: CYP3A4*18, CYP3A5*3, and MDR1-3435 in 133 donors and 133 recipients after living donor liver transplantation

\begin{tabular}{|c|c|c|c|c|c|c|c|c|}
\hline \multirow{2}{*}{$\begin{array}{l}\text { CYP2C19 } \\
\text { Genotype (n) }\end{array}$} & \multicolumn{2}{|c|}{ CYP $3 A 4^{*} 18^{\mathrm{a}}$} & \multicolumn{3}{|c|}{$C Y P 3 A 5^{*} 3^{b}$} & \multicolumn{3}{|c|}{$M D R 1-3435^{c}$} \\
\hline & $\mathrm{T} / \mathrm{T}$ & $\mathrm{T} / \mathrm{C}$ & $\mathrm{G} / \mathrm{G}$ & $A / G$ & $\mathrm{~A} / \mathrm{A}$ & $\mathrm{C} / \mathrm{C}$ & $\mathrm{C} / \mathrm{T}$ & $\mathrm{T} / \mathrm{T}$ \\
\hline HomEM: $\mathrm{n}=102(\%)$ & $93(91.2)$ & $9(8.8)$ & $55(53.9)$ & $35(34.3)$ & $12(11.8)$ & $39(38.2)$ & $51(50.0)$ & $12(11.8)$ \\
\hline$D: n=56(\%)$ & $50(53.8)$ & $6(66.7)$ & $32(58)$ & $17(48.6)$ & $7(58.3)$ & $23(59)$ & $29(56.9)$ & $4(33.3)$ \\
\hline $\mathrm{R}: \mathrm{n}=46(\%)$ & $43(46.2)$ & $3(33.3)$ & $23(42)$ & $18(51.4)$ & $5(41.7)$ & $16(41)$ & $22(43.1)$ & $8(66.7)$ \\
\hline HetEM: $n=130(\%)$ & $127(97.7)^{d}$ & $3(2.3)^{\mathrm{d} \#}$ & $65(50.0)$ & $54(41.5)$ & $11(8.5)$ & $52(40)$ & $64(49.2)$ & $14(10.8)$ \\
\hline D: $n=58(\%)$ & $58(45.7)$ & $0(0)$ & $30(46.2)$ & $26(48)$ & $2(18.2)$ & $22(42.3)$ & $32(50)$ & $4(28.6)$ \\
\hline$R: n=72(\%)$ & $69(54.3)$ & $3(100)$ & $35(53.8)$ & $28(52)$ & $9(81.8)$ & $30(57.7)$ & $32(50)$ & $10(71.4)$ \\
\hline PM: $n=34(\%)$ & $30(88.2)^{d}$ & $4(11.8)^{d}$ & $14(41.2)$ & $20(58.8)$ & & $16(47.1)$ & $16(47.1)$ & $2(5.9)$ \\
\hline D: $n=19(\%)$ & $16(53.3)$ & $3(75)$ & $9(64.3)$ & $10(50)$ & 0 & $11(68.8)$ & $6(37.5)$ & $2(100)$ \\
\hline$R: n=15(\%)$ & $14(46.7)$ & $1(25)$ & $5(35.7)$ & $10(50)$ & & $5(31.2)$ & $10(62.5)$ & $0(0)$ \\
\hline
\end{tabular}

D, donor; HetEM, heterozygous extensive metabolizers; HomEM, homozygous extensive metabolizers; PM, poor metabolizers; R: recipient.; ${ }^{2} C Y P 3 A 4{ }^{*} 18$ (exon 10; T878C).

${ }^{\mathrm{b}}$ CYP3A5*3 (intron 3; A6986G).

${ }^{\mathrm{C}} M D R 1-3435$ (exon 26; C3435T)

${ }^{d} P<0.05$. There was no statistically significant difference between the haplotypes of CYP3A4*18 (T/T and T/C), CYP3A5*3 (G/G, A/G, and A/A), and MDR1-3435 (C/C, $C / T$, and $T / T$ ) and also between the different CYP2C19 genotypes (HomEM, HetEM, and PM) between healthy donors and recipients with end stage liver disease. There were independent isoenzymes and no correlation of genetic interaction between CYP2C19 and CYP3A4*18, CYP3A5*3, or MDR1-3435 not only, but also the variant haplotypes or genotypes. 
identified among individuals with PMs: 64.3\% (9/14) of individuals with the G/G genotype were donors and $35.7 \%$ (5/14) were recipients, and $50.0 \%(10 / 20)$ of individuals with the A/G genotype were donors and 50.0\% (10/20) were recipients. No CYP3A5*3 A/A genotypes were detected. The 3 MDR1-3435 genotypes were distributed as follows: $68.8 \%(11 / 16)$ of individuals with the $\mathrm{C} / \mathrm{C}$ genotype were donors and $31.2 \%(5 / 16)$ were recipients, $37.5 \%$ (6/16) of individuals with the C/T genotype were donors and $62.5 \%(10 / 16)$ were recipients, and $100.0 \%$ $(2 / 2)$ of individuals with the $\mathrm{T} / \mathrm{T}$ genotype were donors. No statistically significant association was found between healthy donors and diseased recipients either with the CYP2C19 PM genotype or with the CYP3A4*18, CYP3A5*3, and MDR1-3435 genotypes $(P>0.05)$.

\section{Discussion}

From our previous studies, we know that CYP2C19 expresses three genotypes with different drug metabolization capacities $[1,3]$. In this study, we attempted to investigate possible genetic interactions between $C Y P 2 C 19$ and CYP3A4*18, CYP3A5*3, or MDR1-3435 in detail. In the present study, we focused on the expression of genetic polymorphisms in the CYP $3 A 4 \% 18, C Y P 3 A 5 \% 3$, and MDR1-3435 genotypes. No significant differences were found in the distributions between CYP3A4*18 (exon 10; T878C), CYP3A5*3 (intron 3; A6986G), or MDR13435 (exon 26; C3435T) genotypes on the basis of different CYP2C19 genotypes between healthy liver donors and patients with poor liver function who received LDLT. Although all these proteins are important isoenzymes of cytochrome P450 in drug metabolism in the liver, only the differences in CYP2C19 genotypes have been documented [1]. CYP3A4*18, CYP3A5*3, and MDR1-3435 polymorphisms do not appear to have a functional effect following LDLT in either the donor with normal liver function or the recipient with ESLD. A recent study showed that the CYP2C19 genotype, unlike MDR1 and $I L-1 B$ genotypes, had an impact on the efficacy of Helicobacter pylori eradication in peptic ulcer patients treated with pantoprazole in triple therapy administrations [7]. In the present study, individuals receiving LDLT who had different CYP2C19 genotypes (HomEM [41 and 43.1\%], HetEM [57.7 and 50\%], and PM [31.2 and 62.5\%]) did not show different distributions of CYP3A4*18,CYP3A5*3, and MDR1-3435 genotypes $(\mathrm{C} / \mathrm{C}$ and $\mathrm{C} / \mathrm{T})$. This observation was consistent in both donors and recipients.

After LDLT, the serum levels of immunosuppressive agents were significantly lower on D1 than on D30, whereas the results of all liver functional tests (ALT, AST, T-Bil, PT(INR), and Alb) were significantly higher on D1 than on D30. These results led us to hypothesize that the immunosuppressive agents affected the stability of metabolic enzymes in the cytochrome P450 system. Previous published reports describe CYP3A as the most abundant enzyme of the P450 subfamily in the human liver and intestine, accounting for $30 \%$ of the total P450 in the human liver, and metabolizing approximately $50 \%$ of currently used clinical drugs [8-10]. The impacts of different CYP2C19, CYP3A4*18, CYP3A5*3, and MDR13435 genotypes on LDLT have been outlined in a flow chart presented in Figure 1. In our previous study, the homogenous phenomenon was attributed to the different CYP2C19 genotypes (HomEM, HetEM, and PM) between an LDLT donor and recipient owing to the uniqueness of the human liver [4]. The metabolism of the proton pump inhibitor (PPI) was dependent on the CYP2C19 genotypes in the cytochrome P450 system, primarily in the liver. HomEM genotypes were found to better metabolize some drugs than did the HetEM and PM genotypes during LDLT [1]. If the donor possessed a CYP2C19 PM genotype, the recipient assumed a PM genotype (rather than

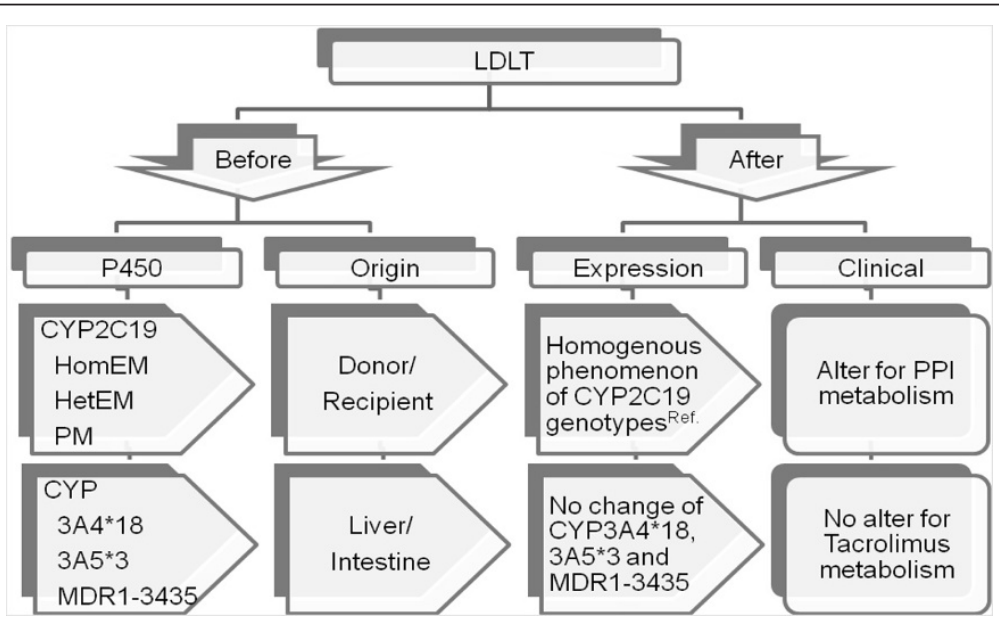

Figure 1 Flow chart of the possible relationship between CYP2C19 genotypes and CYP3A4*18, CYP3A5*3, and MDR1-3435 genotypes. [4]. 
the original CYP2C19 HomEM genotype) because of the homogenous phenomenon [4]. In contrast, the CYP3A4"18 and CYP $3 A 5 * 3$ genetic polymorphisms have two origins: the liver and intestine $[8,9]$. In the postnatal human liver, CYP3A4 and CYP3A5 are the two major CYP3A enzymes, which have overlapping substrate specificities [10]. When the liver function worsens, the drug metabolic function of the intestine may compensate, leading to unaltered drug metabolism, such as for tacrolimus (Figure 1). Depending on the liver function, the CYP2C19 genotypes HomEM, HetEM, and PM were more likely to present abnormal postoperative liver function and graft pathology [3]; however, this was not observed in the present study for the CYP3A4*18, CYP3A5*3, and MDR1-3435 polymorphisms in LDLT. As reported previously, CYP2C19 genotype expression is not demonstrated well by western blotting [11]. CYP3A5 protein expression is highly variable in the human liver, in particular, because of the high frequency of a SNP CYP3A5*3 A6986G in an intron [12]. The CYP3A4*18B SNP in intron 10 was first discovered by direct sequencing in a Japanese population. It was speculated that this variant was associated with increased CYP3A4 activity [5], and this speculation was extended by exploring cyclosporin A (CsA) metabolism in healthy Chinese subjects [6]. A number of SNPs have been identified in the MDR1 gene by large-scale sequencing. For example, our study probed the C3435T variant in exon $26[13,14]$. The PCR-RFLP method to detect the MDR1 3435C/T polymorphism has also been widely used, as was used recently in our study [7]. Although there is no evidence suggesting that intestinal expression of CYP3A4, CYP3A5, and MDR-1 play an important role, human cytochrome $\mathrm{P} 450$ enzymes have been expressed in Escherichia coli. Simplified bacterial systems can explain the possibility of intestinal activation of these enzymes [15]. A previous study has shown that the intestinal mucosa contains prominent forms of cytochrome P450, which are similar to liver cytochrome $\mathrm{P} 450 \mathrm{p}$ in their structure, function, and some regulatory characteristics [16].

Therefore, the results of the present study do not impel us to change strategies and to combine certain donors with certain recipients. According to our previous study, evidence for graft rejection occurred within a month after LDLT [2]. In other words, the cytochrome P450 system stabilizes with time, up to 1 month after LDLT. The increase in serum level of immunosuppressive agents is followed by clinical liver functions leading to stability. Whether these studies can be performed in cadaveric liver transplantation cases is presently unclear.

In our cohort, $68.4 \%$ of individuals receiving LDLT had underlying chronic viral hepatitis-related ESLD, including $39.9 \%$ of individuals who had HCC. There was no difference in CYP3A4*18, CYP3A5*3, and MDR13435 genotypes between the donors and recipients. In addition to the etiology of the underlying disease, age also did not influence the distribution of CYP $3 A 4 * 18$, CYP3A5*3, and MDR1-3435 genotypes. No remarkable difference was observed between donors and recipients, as well as between pediatric and adult recipients.

From our data, the haplotypes of the CYP3A4*18, CYP3A5*3 or MDR1-3435 do not seem to correlate with tacrolimus metabolism in these recipients, but the variant stability of these enzymes are significantly different on D1 and D30 after LDLT. Drug levels were lower on D1 and higher on D30, but there were no correlations to the haplotypes of CYP $3 A 4^{*} 18, C Y P 3 A 5 * 3$, or MDR13435 and/or different genotypes of CYP2C19 HomEM, HetEM, and PM, except for their stability.

In conclusion, the CYP2C19 genotypes, HomEM, HetEM, and PM, do not affect the expression of CYP3A4*18, CYP3A5*3, and MDR1-3435 polymorphisms. These polymorphisms were independently distributed among donors and recipients, as well as healthy and diseased livers, because the source may be located outside the liver during LDLT.

\section{Abbreviations}

Alb: albumin; ALT: alanine transferase; AST: aspartate transferase; DNA: deoxyribonucleic acid; ESLD: end-stage liver disease; HBV: hepatitis B virus; HCC: hepatocellular carcinoma; HCV: hepatitis C virus;

HetEM: heterozygous extensive metabolizer; HomEM: homozygous extensive metabolizer; INR: international normal range; IRB: Institutional Review Board; LDLT: living donor liver transplantation; PCR/LDR: polymerase chain reaction/ ligase detection reaction assay; PCR-RFLP: polymerase chain reactionrestriction fragment length polymorphism; PM: poor metabolizer; PPI: proton pump inhibitor; PT: prothrombin time; SNP: single nucleotide polymorphism; T-Bil: bilirubin total.

\section{Competing interests}

The authors declare that they have no competing interests.

\section{Authors' contributions}

KWC and THH contributed in the preparation of this manuscript in terms of literature review and writing the manuscript. CYL, LWH, HPT, and HCC contributed in data collection, data entry, data analysis, interpretation, and drafting of the article. TN, KDC, YFC, SG, and CLC contributed by writing specific sections of the manuscript and in revising it critically for important intellectual content. All authors have read the manuscript and have approved the final version of this manuscript.

\section{Acknowledgements}

This work was supported by a grant from Chang Gung Memorial Hospital (CMRPG8A0631 to K-WC) of Taiwan. We thank Miss Ching-Yin Huang for her help with data collection.

Received: 14 November 2012 Accepted: 11 April 2013

Published: 23 April 2013

\section{References}

1. Chiu KW, Tai WC, Nakano T, Tseng HP, Cheng YF, Jawan B, Eng HL, Goto S, Chen CL: Donor graft does not affect P450 2C19 genotype expressed on peripheral blood in recipient with living donor liver transplantation. Clin Transplant 2010, 24:830-834.

2. Chiu KW, Chen YS, de Villa VH, Wang CC, Eng HL, Wang SH, Liu PP, Jawan B, Huang TL, Cheng YF, Chen CL: Characterization of liver enzymes on living related liver transplantation patients with acute rejection. Hepatogastroenterology 2005, 52:1825-1827. 
3. Chiu KW, Nakano T, Hu TH, Tseng HP, Cheng YF, Jawan B, Eng HL, Goto S, Chen CL: CYP2C19 genotypes and graft pathology in recipients after liver transplantation. Ann Transpl 2010, 15:38-43.

4. Chiu KW, Nakano T, Hu TH, Tseng HP, Cheng YF, Jawan B, Eng HL, Goto S, Chen CL: Homogenous phenomenon of graft liver CYP2C19 genotypes after living donor liver transplantation. Eur J Clin Invest 2012, 42:352-356.

5. Fukushima-Uesaka H, Saito Y, Watanabe H, Shiseki K, Saeki M, Nakamura T, Kurose K, Sai K, Komamura K, Ueno K, Kamakura S, Kitakaze M, Hanai S, Nakajima T, Matsumoto K, Saito H, Goto Y, Kimura H, Katoh M, Sugai K, Minami N, Shirao K, Tamura T, Yamamoto N, Minami H, Ohtsu A, Yoshida T, Saijo N, Kitamura Y, Kamatani N, Ozawa S, Sawada J: Haplotypes of CYP3A4 and their close linkage with CYP3A5 haplotypes in a Japanese population. Hum Mutat 2004, 23:100.

6. Hu YF, Tu JH, Tan ZR, Liu ZQ, Zhou G, He J, Wang D, Zhou HH: Association of CYP3A4*18B polymorphisms with the pharmacokinetics of cyclosporine in healthy subjects. Xenobiotica 2007, 37:315-327.

7. Gawrońska-Szklarz B, Siuda A, Kurzawski M, Bielicki D, Marlicz W, Droździk M: Effects of CYP2C19, MDR1, and interleukin 1-B gene variants on the eradication rate of Helicobacter pylori infection by triple therapy with pantoprazole, amoxicillin, and metronidazole. Eur J Clin Pharmacol 2010, 66:681-687.

8. Kolars JC, Schmiedlin-Ren P, Schuetz JD, Fang C, Watkins PB: Identification of rifampin-inducible P450IIIA4 (CYP3A4) in human small bowel enterocytes. J Clin Invest 1992, 90:1871-1878.

9. Hughes SJ, Morse MA, Weghorst CM, Kim H, Watkins PB, Guengerich FP, Orringer MB, Beer DG: Cytochromes P450 are expressed in proliferating cells in Barrett's metaplasia. Neoplasia 1999, 1:145-153.

10. Yamazaki H, Shibata A, Suzuki M, Nakajima M, Shimada N, Guengerich FP, Yokoi T: Oxidation of troglitazone to a quinone-type metabolite catalyzed by cytochrome P-450 2C8 and P-450 3A4 in human liver microsomes. Drug Metab Dispos 1999, 27:1260-1266.

11. Chiu KW, Nakano T, Tseng HP, Cheng YF, Jawan B, Eng HL, Goto S, Chen CL: CYP2C19 activity of liver tissues in western blot analysis on living donor liver transplantation. Hepatogastroenterology 2012, 59:805-808.

12. Kuehl P, Zhang J, Lin Y, Lamba J, Assem M, Schuetz J, Watkins PB, Daly A, Wrighton SA, Hall SD, Maurel P, Relling M, Brimer C, Yasuda K, Venkataramanan R, Strom S, Thummel K, Boguski MS, Schuetz E: Sequence diversity in CYP3A promoters and characterization of the genetic basis of polymorphic CYP3A5 expression. Nat Genet 2001, 27:383-391.

13. Hoffmeyer S, Burk O, von Richter O, Arnold HP, Brockmoller J, Johne A, Cascorbi I, Gerloff T, Roots I, Eichelbaum M, Brinkmann U: Functional polymorphisms of the human multidrug-resistance gene: multiple sequence variations and correlation of one allele with P-glycoprotein expression and activity in vivo. Proc Natl Acad Sci USA 2000, 97:3473-3478.

14. Cascorbi I, Gerloff T, Johne A, Meisel C, Hoffmeyer S, Schwab M, Schaeffeler E, Eichelbaum M, Brinkmann U, Roots I: Frequency of single nucleotide polymorphisms in the P-glycoprotein drug transporter MDR1 gene in white subjects. Clin Pharmacol Ther 2001, 69:169-174.

15. Shimada T, Gillam EM, Sandhu P, Guo Z, Tukey RH, Guengerich FP: Activation of procarcinogens by human cytochrome P450 enzymes expressed in Escherichia coli. Simplified bacterial systems for genotoxicity assays. Carcinogenesis 1994, 15:2523-2529.

16. Watkins PB, Wrighton SA, Schuetz EG, Molowa DT, Guzelian PS: Identification of glucocorticoid-inducible cytochromes P-450 in the intestinal mucosa of rats and man. J Clin Invest 1987 Oct, 80:1029-1036.

doi:10.1186/2047-1440-2-6

Cite this article as: Chiu et al: Biological interactions of CYP2C19 genotypes with CYP3A4*18, CYP3A5*3, and MDR1-3435 in living donor liver transplantation recipients. Transplantation Research 2013 2:6.

\section{Submit your next manuscript to BioMed Central and take full advantage of:}

- Convenient online submission

- Thorough peer review

- No space constraints or color figure charges

- Immediate publication on acceptance

- Inclusion in PubMed, CAS, Scopus and Google Scholar

- Research which is freely available for redistribution

Submit your manuscript at www.biomedcentral.com/submit 